\title{
Graph-based Implementation of a Functional Logic Language*
}

\author{
Herbert Kuchen, Rita Loogen \\ RWTH Aachen ${ }^{\dagger}$
}

\author{
Juan José Moreno-Navarro \\ Universidad Politécnica de Madrid ${ }^{\ddagger}$
}

\author{
Mario Rodríguez-Artalejo. \\ Universidad Complutense de Madrid ${ }^{\S}$
}

\begin{abstract}
We present in this paper a graph-narrowing abstract machine which has been designed to support a sequential eager implementation of a functional logic language. Our approach has been to extend a purely functional, (programmed) graph reduction machine by mechanisms capable of performing unification and backtracking. We describe the structure of the machine and explain the compilation scheme which generates machine code from a given source program. Both the machine and the compilation scheme have been formally specified. A prototype emulator of the machine has been implemented in Occam on a transputer system. Future work is planned for incorporating lazy evaluation and parallelism to the machine.
\end{abstract}

\section{Introduction}

During the last years, several approaches have been proposed to achieve an integration of functional and logic programming languages in order to combine the advantages of the two main declarative programming paradigms in a single framework [DeGroot, Lindstrom 86], [Bellia, Levi 86]. Usually one argues that logic languages have more expressive power than functional languages, while the latter have a simpler execution model, particularly suited to parallel implementations.

Roughly, many of the existing approaches to the integration of functional and logic programming can be partitioned into two classes: On the one hand, Horn clause logic languages enhanced with equality and functions; on the other hand, functional languages augmented with logical capabilities. This second class includes the so called functional logic languages [Reddy 85,87], which retain functional syntax but use narrowing - a unification based parameter passing mechanism which subsumes reduction and SLD-resolution - as operational semantics.

We investigate in this paper a sequential, eager implementation of the functional logic language BABEL [Moreno, Rodríguez 89] on a graph narrowing abstract machine. Up to now, BABEL had been developped as a first order, type free language. Here, we extend it to a higher order functional logic language with polymorphic typing.

Our approach has been to extend the sequential kernel of a parallel (programmed) graph reduction machine which has been designed for the execution of functional programming languages [Loogen et al. 89] by the additional features that are necessary to execute functional logic programs, namely,

\footnotetext{
*This work has been partially supported by a german-spanish cooperation action, funded by the german D.A.A.D. and the spanish M.E.C

${ }^{\dagger}$ Lehrstuhl für Informatik II, Ahornstraße 55, 5100 Aachen, West Germany

†Departarnento de Lenguajes y Sistemas Informáticos e Ingeniería de Software, Facultad de Informática, Campus de Montegancedo, Boadilla del Monte, 28660 Madrid, Spain

§Departamento de Informática y Automática, Facultad de C.C. Matemáticas, 28040 Madrid, Spain
} 
unification and backtracking facilities. For simplicity, we discuss in this paper the realization of an innermost narrowing strategy, i.e. the arguments of a function call are evaluated before the function is applied. However, we have chosen a graph reduction model because our final aim is a distributed parallel machine supporting lazy evaluation. A prototype emulator of the new abstract machine has been programmed in Occam and runs on transputer systems.

A distinctive feature of our approach is that SLD-resolution is subsumed by narrowing, which becomes the single inference mechanism of the language and works both for the Prolog-like part and for the purely functional part. As shown by several researchers [van Emden, Yukawa 87], [Bosco et al. 88], narrowing can be also subsumed by SLD-resolution. Several integrated logic plus functional programming languages - which follow a Horn clause logic based approach for the integration have chosen some modified form of SLD-resolution as an implementation basis, in order to capitalize on the extensive experience already available for Prolog implementations. The language K-LEAF [Levi et al. 87], [Bosco et al. 89] is a prominent example of this. We have chosen our approach by similar reasons, namely, in order to profit from the available experience in implementation techniques for functional languages. We hope that purely applicative programs will run on the BABEL machine almost as efficiently as in the original graph reduction machine, although some overhead due to the different parameter passing mechanisms cannot be avoided. The narrowing based approach has also the advantage of being conceptually very clear and simple.

This paper is organized as follows. In section 2 we give a short presentation of the language BABEL. The structure of the BABEL abstract machine is described in section 3 , where we also explain the compilation of BABEL programs into code for the abstract machine. The compilation rules are very similar to the functional case, because backtracking is not controlled by code, but is instead performed by an implicit mechanism which is started by a special machine instruction. Formal specifications of both the machine and the compilation scheme can be found in [Kuchen et al. 89]. In section 4 we explain in more detail the organization of backtracking, which is the most important novel feature of the machine, since machine instructions for unification are borrowed from Warren's abstract machine for Prolog [Warren 83]. In section 5 we report on the current state of the implementation, which includes some optimizations. Section 6 includes some comments on related works. In section 7 we recapitulate our results and report on some planned future works.

\section{The functional logic language BABEL}

The language BABEL has been designed by Mario Rodríguez-Artalejo and Juan José Moreno-Navarro to achieve integration of functional and logic programming in a flexible and mathematically wellfounded way [Moreno, Rodríguez 88,89], [Moreno 89]. It is based on a constructor discipline and uses narrowing as an evaluation mechanism; cfr. [Reddy 85,87]. In this paper we work with an extension of BABEL that supports polymorphic types and higher-order functions. However, higher-order logic variables are not allowed, i.e. higher-order variables are never affected by narrowing.

Before we give a formal presentation of BABEL-programs, we consider a small example program:

$$
\begin{aligned}
& \text { typevar } A, B \text {. } \\
& \text { type } \quad \operatorname{list}(A)=\text { nil } \mid \text { cons }(A, \operatorname{list}(A)) \text {. } \\
& \text { fun map: }(A \rightarrow B) \rightarrow \operatorname{list}(A) \rightarrow \operatorname{list}(B) \text {. } \\
& \quad \operatorname{map} F[]:=[] \text {. } \\
& \quad \operatorname{map} F[X \mid X s]:=[F X \mid \operatorname{map} F X s] \text {. } \\
& \text { eval }(\operatorname{map}(+2)[X, 3])=[6, Y] \text {. }
\end{aligned}
$$

that will yield the boolean value true with variable bindings $\{X / 4, Y / 5\}$.

Note that we assume numbers and arithmetic operations as well as the equality operator to be predefined. We also allow a PROLOG-like syntax for lists.

The higher-order function map can be used in a very flexible way due to the first-order logic variables. The higher-order variable $\mathrm{F}$ can only be used as in applicative programming. 


\subsection{Syntax}

Let $\Sigma=\left\langle T C_{\Sigma}, D C_{\Sigma}, F S_{\Sigma}\right\rangle$ be a polymorphic signature, i.e.

- $T C_{\Sigma}$ is a set of ranked type constructors : tc/n, e.g. nat $/ 0$, list $/ 1, \ldots$

- $D C_{\Sigma}$ is a set of typed data constructors: $c: \tau, c^{\prime}: \tau_{1} \times \ldots \times \tau_{n} \rightarrow \tau$ with $\tau=\operatorname{tc} / m\left(\alpha_{1}, \ldots, \alpha_{m}\right)$ and $r_{i} \in \mathrm{CType}_{\Sigma}$, where CType , $_{\Sigma}$ is the set of constructed types over type variables $\alpha_{1}, \alpha_{2} \ldots \in$ $\operatorname{TVar}_{\Sigma}$ defined recursively by

$$
\tau::=\alpha|\mathrm{tc} / 0| \mathrm{tc} / n\left(\tau_{1}, \ldots, \tau_{n}\right) \% n \geq 2
$$

and the type variables in $\tau_{1}, \ldots, \tau_{n}$ are taken from $\left\{\alpha_{1}, \ldots, \alpha_{m}\right\}$.

- $F S_{\Sigma}$ is the set of typed function symbols $f: \tau$ with $\tau \in \mathbf{T y p e}_{\Sigma}$, where $\mathbf{T y p e}_{\Sigma}$ is the set of polymorphic types defined by

$$
\tau::=\alpha|\mathrm{tc} / 0| \mathrm{tc} / n\left(\tau_{1}, \ldots, \tau_{n}\right) \mid\left(\tau \rightarrow \tau^{\prime}\right) .
$$

In the sequel we assume that " $\rightarrow$ " associates to the right and omit brackets accordingly.

We assume that $\Sigma$ contains the predefined types:

- bool with data constructors true, false and function symbols $\neg:$ bool $\rightarrow$ bool (logic negation), $\Lambda:$ bool $\rightarrow$ bool $\rightarrow$ bool (sequential and), $\vee:$ bool $\rightarrow$ bool $\rightarrow$ bool (sequential or) and

- nat with data constructors 0 and $s$ (successor function) and the usual arithmetic functions.

A special primitive function symbol is the weak equality symbol $=$ with type $\alpha \rightarrow \alpha \rightarrow$ bool whose definition will be given later.

In example programs we declare constructed types and data constructors in a MIRANDA-like style, cfr. [Turner 85].

We distinguish the following syntactic domains:

- variables ranged over by $\mathrm{X}, \mathrm{X}, \mathrm{Z} \ldots \in \mathrm{Var}$,

- terms ranged over by $s, t \ldots \in$ Term $_{\Sigma}$ :

$$
\begin{aligned}
& \begin{array}{rll}
t::= & \% \text { variable } \\
\mid c & \% \text { constant (nullary constructor, } c / 0 \in D C_{\Sigma} \text { ) }
\end{array} \\
& \text { | } c\left(t_{1}, \ldots, t_{n}\right) \% c / n \in D C_{\Sigma} \text {, construction, respecting types }
\end{aligned}
$$

- expressions ranged over by $\mathrm{B}, \mathrm{C}, \mathrm{M}, \mathrm{N} \ldots \in \mathbf{E x p}_{\Sigma}$

$$
M::=\begin{array}{ll}
t & \text { \% term } \\
c\left(M_{1}, \ldots, M_{n}\right) & \% c / n \in D C_{\Sigma}, \text { construction, respecting types } \\
f & \text { \% } f \in F S_{\Sigma} \text {, function symbol } \\
(M N) & \text { \% application, respecting types } \\
(B \rightarrow M) & \text { \% guarded expression, } B: \text { bool } \\
\left(B \rightarrow M_{1} \square M_{2}\right) & \text { \% conditional expression, } B: \text { bool, } M_{1}, M_{2}: \tau \text { for some } \tau
\end{array}
$$

Expressions should be well-typed. We omit the formal definition of a type inference system for expressions; cfr. [Milner 78, Damas, Milner 82]. In the sequel we will reserve $B, C$ for boolean expressions.

We remark that $B \rightarrow M$ and $B \rightarrow M_{1} \square M_{2}$ are intended to mean "if $B$ then $M$ else undefined" and "if $B$ then $M_{1}$ else $M_{2}$," respectively.

We shall assume that application associates to the left and omit brackets accordingly. 
A BABEL-program of signature $\Sigma$ consists of a set of defining rules for the non predefined symbols in $F S_{\Sigma}$. The rules for the predefined symbols are implicitly added to every program, and will be presented later.

Notice that any $f \in F S_{\Sigma}$ must have type $\tau_{1} \rightarrow \ldots \rightarrow \tau_{n} \rightarrow \tau$, for some $n \geq 0$ and some $\tau$ that is not of the form $\tau^{\prime} \rightarrow \tau^{\prime \prime}$. Here, $n$ is the type-arity of $f$.

Each defining rule for $f$ must have the form

$$
\underbrace{f t_{1} \ldots t_{m}}_{\text {lhs }}:=\underbrace{\{B \rightarrow\}}_{\text {rhs }} \underbrace{M}_{\begin{array}{c}
\text { guard } \\
\text { (optional) }
\end{array}}
$$

for some $m \leq n$ (called the arity of the rule) and satisfy the following restrictions:

1. Flatness: $t_{i} \in \operatorname{Term}_{\Sigma}$.

2. Left Linearity: $f t_{1} \ldots t_{n}$ does not contain multiple variable occurrences.

3. Well-Typedness: Under appropriate type assumptions for the variables we may infer the types $\tau_{i}$ for the terms $t_{i}(1 \leq i \leq m)$, the type bool for the guard $B$ and the type $\tau_{m+1} \rightarrow \ldots \rightarrow \tau_{n} \rightarrow \tau$ for the body $M$.

4. Restrictions on free variables: Any variable that occurs in the rhs but not in the lhs is called free. Occurrences of free variables are allowed in the guard, but not in the body. Moreover, free variables must be first-order. By this we mean that their types must be constructed types (under the type assumption used to well-type the rule).

In any program, all rules for a fixed $f$ must have the same arity. This is called the program-arity of $f$, and is less or equal than $f$ 's type-arity. Programs are also required to satisfy a nonambiguity condition:

5. Nonambiguity: Given any two rules for the same function symbol $f$ :

$$
\begin{aligned}
& f t_{1} \ldots t_{m}:=\{B \rightarrow\} M \\
& f s_{1} \ldots s_{m}:=\{C \rightarrow\} N
\end{aligned}
$$

one of the three following cases must hold:

(a) No superposition: $f t_{1} \ldots t_{m}$ and $f s_{1} \ldots s_{m}$ are not unifiable.

(b) Fusion of bodies: $f t_{1} \ldots t_{m}$ and $f s_{1} \ldots s_{m}$ have a most general unifier (m.g.u.) $\sigma$ such that $M \sigma, N \sigma$ are identical ${ }^{1}$.

(c) Incompatibility of guards: $f t_{1} \ldots t_{m}$ and $f s_{1} \ldots s_{m}$ have a m.g.u. $\sigma$ such that $(B \wedge C) \sigma$ is unsolvable.

This depends on a notion of unsolvability that must be chosen decidable and such that unsolvable boolean expressions cannot yield the value true under any valuation of their variables; $\mathrm{cfr}$. [Moreno, Rodríguez 89].

At this point, a short digression on the nonambiguity condition seems appropriate. Some designers of integrated logic plus functional languages, e.g. K-LEAF [Levi et al. 87], have adopted similar nonambiguity conditions in order to guarantee confluence, which in turn is generally believed to be a necessary condition for the completeness of narrowing as an equation solving procedure, cfr. [Hullot 80]. Actually, it is possible to dispose of confluence, provided that the language's semantics is suitably changed to accommodate a notion of nondeterministic function. Nevertheless, some subtle

\footnotetext{
${ }^{1}$ As usual, $M \sigma$ denotes the expression $M$ where all variables are replaced according to $\sigma$.
} 
semantic problems must be solved in this approach; see [Hussmann 89]. For simplicity, we have chosen to avoid ambiguity in our presentation, though the BABEL machine is able to execute ambiguous programs by backtracking.

We assume some predefined rules for the primitive function symbols and the guarded and conditional expressions. Among them, we have

- Rules for the boolean operations

$$
\begin{array}{lll|}
\neg \text { false } & := & \text { true } \\
\neg \text { true } & := & \text { false }
\end{array}
$$

\begin{tabular}{lll|}
\hline false $\wedge \mathrm{Y}$ & $:=$ & false \\
true $\wedge \mathrm{Y}$ & $:=$ & $\mathrm{Y}$ \\
\hline
\end{tabular}

\begin{tabular}{lll|}
\hline false $\vee Y$ & $:=$ & $Y$ \\
true $\vee Y$ & $:=$ & true \\
\hline
\end{tabular}

- Rules for weak equality

$$
\begin{aligned}
& (c=c):=\text { true } \\
& \left(c\left(X_{1}, \ldots, X_{n}\right)=c\left(Y_{1}, \ldots, Y_{n}\right)\right):=\left(X_{1}=Y_{1}\right) \wedge \ldots \wedge\left(C_{\Sigma},\right. \text { constant } \\
& \left(c\left(X_{1}, \ldots, X_{n}\right)=d\left(Y_{1}, \ldots, Y_{m}\right)\right):=\text { false } \% c / n \in D C_{\Sigma}, d / m \in D C_{\Sigma} \text { different }
\end{aligned}
$$

- Rules for guarded and conditional expressions

$$
(\text { true } \rightarrow X):=X
$$

$$
\begin{array}{|ll|}
(\text { true } \rightarrow X \square Y) & :=X \\
(\text { false } \rightarrow X \square Y) & :=Y \\
\hline
\end{array}
$$

The rules for $\wedge, \vee$ reflect the sequential character of these connectives. The rules for weak equality must be used respecting the types of constructors. They specify that an expression $\left(M_{1}=M_{2}\right)$ will evaluate to true if $M_{1}, M_{2}$ evaluate both to the same term, and will evaluate to false if $M_{1}, M_{2}$ evaluate to different terms. $\left(M_{1}=M_{2}\right)$ will be undefined if the evaluation of $M_{1}$ or $M_{2}$ does not terminate.

BABEL, as described in [Moreno, Rodríguez 89], supports infinite terms through lazy evaluation. This gives rise to a more sophisticated behaviour of weak equality, since an expression $\left(M_{1}=M_{2}\right)$ may be lazily evaluated to false even if the complete evaluation of $M_{1}$ or $M_{2}$ would not terminate.

As a last remark on BABEL programs, let us mention that pure PROLOG can be straightforwardly translated to BABEL. For instance, the PROLOG program

$$
\begin{aligned}
& \text { append }([], Y s, Y s) \text {. } \\
& \text { append(}([X \mid X s], Y s,[X \mid Z s]):-\operatorname{append}(X s, Y s, Z s) \text {. }
\end{aligned}
$$

can be translated as follows:

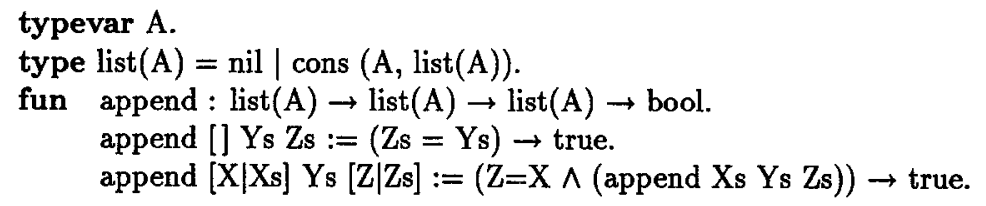

The idea is that PROLOG clauses translate into guarded BABEL rules whose body is identical to true. Weak equality must be used to force left linearity, by adding some new equalities between variables to the guard, if needed. Boolean valued functions play the role of predicates. In fact, a concrete BABEL implementation could allow a PROLOG-like syntax as syntactic sugar; in this way, pure PROLOG programs would be legal BABEL programs almost without any syntactic changes, cfr. [Moreno, Rodríguez 89].

Of course, append can also be programmed as a function in BABEL. The point is that the just given version behaves like the PROLOG append predicate under BABEL's evaluation mechanism. 


\subsection{Narrowing Semantics}

A goal for a given BABEL $\Sigma$-program is any $\Sigma$-expression $M$ which includes no higher-order variables. To solve a goal, the BABEL machine tries to reduce it to a normalized form by means of narrowing. This means that the lhs of rules for the defined and predefined function symbols are unified with appropriate subexpressions, which are then replaced by the corresponding instance of the rule's rhs. This process is repeated until a normal form $N$ is reached. Then, $N$ is taken as the result of the evaluation, and all bindings of variables occurring in $M$ that have been accumulated during the reduction are regarded as the answer, similarly as in PROLOG. The combination of result and answer will be called outcome in the sequel.

The restrictions imposed on higher-order variables are worthy of being noted carefully. Higherorder variables may occur in the lhs of rules, but are forbidden to occur free in either rhs of rules or goals. This means that they are used only for rewriting, as in functional programming.

The narrowing semantics of BABEL is based on the following narrowing rule:

Let $f t_{1} \ldots t_{m}:=R$ be a variant of a BABEL rule in the program which shares no variables with $\left(f M_{1} \ldots M_{m}\right)$. If there exists some most general unifier $\sigma \cup \theta$ (where $\sigma$ binds variables in $\left(f M_{1} \ldots M_{m}\right)$ and $\theta$ binds variables in $\left.\left(f t_{1} \ldots t_{m}\right)\right)$ with

$$
t_{i} \theta=M_{i} \sigma \text { for } 1 \leq i \leq m,
$$

then we may reduce

$$
\left(f M_{1} \ldots M_{m}\right) \longrightarrow_{\theta} R \theta
$$

The one-step narrowing relation

$$
M \Longrightarrow_{\sigma} N
$$

where $M, N$ are BABEL expressions and $\sigma$ is a finite substitution of some first order variables occurring in $M$ by terms, will be defined as follows

- $M_{i} \longrightarrow N_{i}$ with $i \in\{1, \ldots, n\}$ implies

$$
\begin{aligned}
& c\left(M_{1}, \ldots, M_{i}, \ldots, M_{n}\right) \Longrightarrow_{\sigma} c\left(M_{1} \sigma, \ldots, N_{i}, \ldots, M_{n} \sigma\right) \text { and } \\
& \left(M_{1} \ldots M_{i} \ldots M_{n}\right) \Longrightarrow_{\sigma}\left(M_{1} \sigma \ldots N_{i} \ldots M_{n} \sigma\right)
\end{aligned}
$$

- $B \longrightarrow_{\sigma} B^{\prime}$ implies

$$
(B \rightarrow M) \Longrightarrow_{\sigma}\left(B^{\prime} \rightarrow M \sigma\right) \text { and }\left(B \rightarrow M_{1} \square M_{2}\right) \Longrightarrow_{\sigma}\left(B^{\prime} \rightarrow M_{1} \sigma \square M_{2} \sigma\right)
$$

We write

$$
M \stackrel{*}{\Longrightarrow} N
$$

to indicate the result of several narrowing steps, where $\sigma$ is the composition of the substitutions involved in the single steps, restricted to the variables occurring in $M$.

Narrowing of a BABEL-expression with constructed type may have the following outcomes:

- success: $M \stackrel{*}{\Rightarrow} t$ with $t \in$ Term $_{\Sigma}$

- failure: $M \stackrel{*}{\Longrightarrow} N, N$ is not further narrowable and $N \notin T e r m_{\Sigma}$

- nontermination.

For simplicity, we restrict ourselves in this paper to an innermost narrowing strategy. Of course, this implies that infinite objects cannot be used. 
We show the innermost evaluation of a goal for the map program. The redex at each step is underlined, and some intermediate steps (corresponding to predefined functions) are skipped.

$$
\begin{aligned}
& \operatorname{map}(+2)[X, 3]=[6, Y]
\end{aligned}
$$

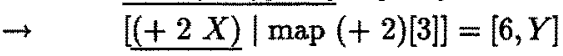

$$
\begin{aligned}
& \rightarrow \quad\left[s^{2}(X) \mid \underline{\operatorname{map}(+2)[3]]}=[6, Y]\right.
\end{aligned}
$$

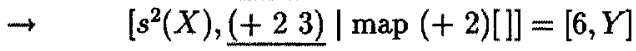

$$
\begin{aligned}
& \rightarrow \quad\left[s^{2}(X), 5 \mid \underline{\operatorname{map}}(+2)[]\right]=[6, Y] \\
& \rightarrow \quad\left[s^{2}(X), 5\right]=[6, Y] \\
& \rightarrow \quad \underline{\left(s^{2}(X)=6\right)} \wedge([5]=[Y]) \\
& \stackrel{*}{\rightarrow}_{\{X / 4\}} \quad \underline{([5]=[Y])} \\
& \rightarrow \quad(\underline{(5=Y)} \wedge([]=[])) \\
& \stackrel{*}{\rightarrow}\{Y / 5\} \quad \text { true }
\end{aligned}
$$

The outcome of this derivation consists of result true and answer $\{X / 4, Y / 5\}$. Some predefined rules for $t,=$ and $\wedge$ have been used.

The BABEL machine tries the program's rules in their textual ordering, tries to evaluate functional expressions before applying them, and evaluates arguments from left to right; it backtracks when a failure or a user's request for alternative solutions occurs.

Primitive symbols are handled as if the user had introduced them through their predefined rules. However, this is not exactly so in all cases: Guarded and conditional expressions are handled in the usual non-strict way, i.e. evaluation of the guard/condition before evaluation of the alternatives. The evaluation of the second member in conjunctions and disjunctions is avoided whenever possible, and weak equality is implemented through unification for the sake of efficiency. This implementation works fine with any equality $t_{1}=t_{2}$ between two terms whose unification either succeeds or finitely fails; but it does not work properly in cases such as $X=c(Y)$, which allow for infinitely many outcomes with result false and different answers, according to the predefined rules of weak equality. The actual implementation will ignore these outcomes. The user can overcome this limitation by explicitly programming his own equality. The price to pay will be a risk of nontermination.

For the first-order fragment of BABEL there is also a declarative semantics, based on algebraic, consistently complete CPOs, and related to a lazy version of narrowing through soundness and completeness theorems; cfr. [Moreno, Rodriguez 89], [Moreno 89]. These results are in the same spirit as those obtained for the language K-LEAF [Levi et al. 87]. Soundness and completeness results are also presented in [Reddy 85] for the eager case, and claimed to hold in [Reddy 87]. However, Reddy's denotational semantics is rather different from our declarative semantics.

\section{Structure of the BABEL Machine}

The BABEL machine is a sequential abstract graph narrowing machine by which the functional logic language BABEL will be implemented using an innermost evaluation strategy. This strategy has been chosen for simplicity in order to develop a first version of an abstract machine for BABEL.

The main component of the machine is a graph, which contains, among others, so called task nodes which correspond to ordinary activation records but contain much more information. A task node contains e.g. a local data stack for data manipulations and a local program counter. For the organization of backtracking a local trail is necessary. It is used in the same way as in the Warren Abstract Machine (WAM) [Warren 83] to keep track of variable bindings which must be removed in the case of backtracking.

Due to the use of local data stacks and local trails the machine has a very decentralized organization. This will simplify a later parallelization of the machine, i.e. the incorporation of the machine in a parallel environment. 


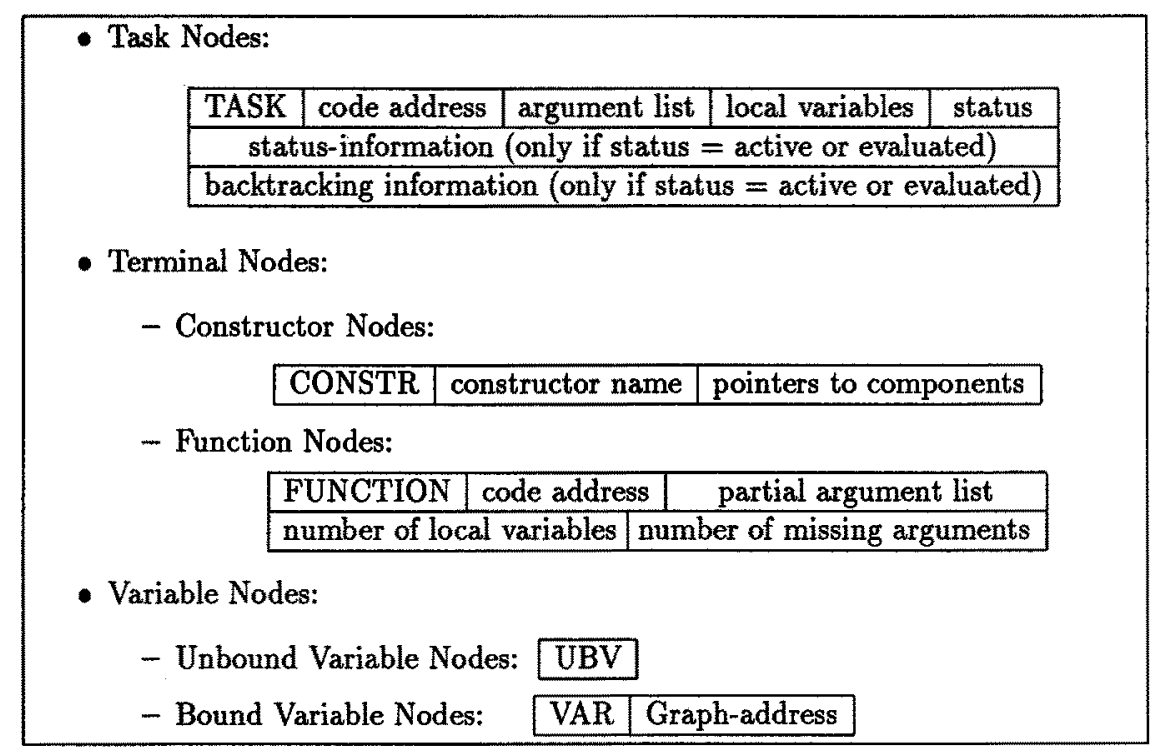

Figure 1: Structure of Graph Nodes

The store of the BABEL machine consists of three components:

- the program store which contains the translations of the BABEL rules into machine code,

- the graph, which may contain task-, variable- and terminal nodes, and

- the active task pointer which points at the task node which corresponds to the currently executed procedure call. A BABEL procedure consists of all program rules corresponding to one function symbol.

Thus, we define

$$
\mathcal{B A M}:=\langle\underbrace{\mathrm{St}}_{\text {Store }}, \underbrace{\vdash}_{\begin{array}{c}
\text { Transition } \\
\text { relation }
\end{array}}\rangle
$$

where

St $:=$ Program_store $\times$ Active_task_pointer $\times$ Graph.

A state of the machine is usually denoted by $(p, a t p, G)$, where $p \in$ Program store, atp $\in$ Active_task_pointer and $\mathbf{G} \in$ Graph.

\subsection{The Graph Component}

The graph component is modelled as a mapping from graph addresses into the graph nodes:

$$
\begin{gathered}
\text { Graph }:=\text { Graph_addresses } \rightarrow \text { Graph_nodes, where } \\
\text { Graph_nodes }:=\text { Task_nodes U Terminal_nodes U Variable_nodes. }
\end{gathered}
$$

Figure 1 indicates the structure of the different graph nodes. The computation is controlled by the task nodes which represent applications.

Each task node contains the address of the first line of code for the corresponding function symbol, pointers to the graph representation of the arguments and a list of pointers to unbound variable nodes which represent local variables. Depending on the status of a task - we distinguish 
dormant, active and evaluated tasks - additional information is provided within the task node. The status-information of active and evaluated tasks consists of a local stack which is needed for the organization of data manipulations, a program counter which indicates the next instruction and a pointer to the father node, i.e. the node by which the current node has been created. This father pointer is used when a task finishes successfully and the control has to be returned to the father task.

Since BABEL is also a logic language, tasks must be able to perform backtracking. For this reason, active and evaluated task nodes contain certain backtracking information. This consists of a local trail which keeps track of variable bindings to be removed in case of backtracking; pointers to the 'previous brother' and the 'last son', which help to find the task to be reactivated when backtracking occurs; a backtrack address, which points to the code that must be executed if backtracking reactivates the task, and finally safe copies of the father's program counter, local stack and local trail, that are needed to restore the machine state in case of backtracking. All this will be explained in detail in section 4 , where the organization of backtracking will be discussed.

In addition to the task nodes the graph contains terminal and variable nodes. Terminal nodes are constructor or function nodes.

Constructor nodes represent structured data. They contain the constructor name and a list of pointers to the graphs of the components of the structure.

Function nodes represent functional data which always correspond to partial function applications. Consequently a function node contains essentially the same base information as a task node (address of first line of code of the function, (partial) list of arguments, number of local variables, i.e. the maximum number of variables occurring in a rule for the function symbol) and additionally the number of arguments that are necessary to make the function application complete.

Variable nodes are needed for the organization of unification. We distinguish nodes for unbound and bound variables. Unbound variable nodes consist only of a tag (UBV). When a variable is bound to some terminal node the graph address of this node is written into the bound variable node which is indicated by the tag VAR. No more information needs to be stored for variables.

\subsection{Machine Instructions}

This subsection gives a short discussion of the machine instructions of $B A M$. Five classes of machine instructions are distinguished.

\subsubsection{Stack Instructions}

Stack instructions are needed for an efficient implementation of some of the primitive functions of $\mathrm{BABEL}$, especially for the logic negation (NOT) and the equality operator (CHECKEQ).

\subsubsection{Graph Instructions}

Graph instructions are used for the graph manipulation. By LOAD-instructions the addresses of arguments of the active task (LOAD i) or pointers to local variables (LOADX i) can be pushed onto the local stack, i.e. the stack within the task node of the active task.

Constructor nodes are generated by execution of the instruction 'CONSTRNODE $(c, m)$ ', where $c$ is the constructor name and $m$ is the number of components whose addresses are taken from the local stack and replaced by the address of the new node.

Task and function nodes are created by the instruction 'NODE (ca, numarg, arity, locals)', which has four parameters: the code address of the function, the number of arguments of the function application that are given on the stack, the program arity of the function, and finally the number of local variables. If enough arguments are given, i.e. numarg = arity, a dormant task node will be constructed. Otherwise, a function node will be created.

To add further arguments to a function node, we use the instruction APPLY $i$ that expects $i$ graph addresses and a pointer to a function node on top of the stack. The $i$ graph addresses are 
added to the argument list of the function node. If this yields a complete application, a dormant task node is generated. Otherwise, a new function node is built. The existing function node must not be overwritten because of the possibility of sharing.

\subsubsection{Unification Instructions}

For the organization of unification we need the following instructions.

'UNIFYCONST (c, arity, label)' tries to unify the top element of the local stack with the constructor $\mathrm{c}$. The top element of the data stack points at a constructor node or at a variable node.

In the case of a constructor node with constructor $c$ the argument list of this node is copied onto the stack. Further unification steps will be controlled by the code that follows the UNIFYCONSTinstruction. If the constructor name in the node is different from $\mathrm{c}$ backtracking occurs.

If the top of the stack points at an unbound variable node, this variable must be bound to a term whose top level constructor will be $c$. In this case a graph representation for the term must be constructed. The address of the code that generates such a graph representation is given as the third parameter (label) of the UNIFYCONST-instruction. Thus in the case of an unbound variable the program counter of the active task is set to 'label'. After the construction of the graph the binding of the unbound variable to the new graph is performed by executing the instruction BIND, that expects a pointer to an unbound variable node and a pointer to a constructor node on top of the stack and that binds the variable to the term graph whose root is the constructor node.

The 'UNIFYVAR $i$ '-instruction binds the $i$-th local variable, that will be unbound when executing this instruction due to the linearity restriction of the BABEL-rules, to the graph whose address is on top of the stack. The implementation of an occur-check and a general unification algorithm is not necessary at this place due to the left linearity.

The UNDO-instruction is used to delete variable bindings in case of backtracking.

\subsubsection{Control Instructions}

Control instructions are jump instructions. The unconditional 'JMP label' sets the program counter of the active task to label. The conditional jump instructions 'JMT label' and 'JMF label' cause a jump only if the boolean value true and false, respectively, is represented by the top element of the stack.

\subsubsection{Process Instructions}

The activation and termination of tasks is controlled by the process instructions EVALUATE and RET, respectively. The EVALUATE-instruction performs a subroutine call to the dormant task whose address is given on top of the stack. The RET-instruction is executed when a task terminates successfully and the control can be given back to the father task.

For the organization of backtracking the instructions 'BACKTRACK label' and 'FAILRET' are necessary. The BACKTRACK-instruction initializes the backtracking information of a task. FAILRET will be executed when a task fails, i.e. no solution can be produced. The 'predecessor' of the task must then be reactivated and forced to evaluate in a different way. For more details, see the explanation of backtracking in section 4 .

To control the behaviour of the machine on the top level, some more instructions are necessary.

- The instruction 'MORE' asks the user if more solutions are to be searched.

- The instruction 'FORCE' forces the last successfully terminated task to backtrack and thus to compute more solutions.

- The instruction 'PRINTFAILURE' finishes the whole execution with the output 'no (more) solutions have been found'. The instruction 'PRINTRESULT' is used to output a solution which consists of the result value and bindings of the local variables within the objective. 


\subsection{Compilation of BABEL-Programs}

A BABEL program consists (mainly) of a set of rules and an expression (called the objective or goal), which is to be evaluated using the rules. The rules are grouped according to the function symbol they define. Hence, a BABEL program looks like this:

$$
\begin{aligned}
& \operatorname{PROC}\left(f_{1}, m_{1}, k_{1}\right) \\
& \ldots \\
& \operatorname{PROC}\left(f_{n}, m_{n}, k_{n}\right) \\
& \text { OBJECTIVE }\left(k_{0}\right)
\end{aligned}
$$

where $\operatorname{PROC}\left(f_{i}, m_{i}, k_{i}\right)$ denotes the set of rules defining function symbol $f_{i}$ with program-arity $m_{i}$ and $k_{i}$ local variables $(1 \leq i \leq n) . k_{0}$ is the number of variables within the objective. The machine code for a BABEL program is the following:

$$
\begin{aligned}
0: & \text { NODE }\left(\text { obj, } 0,0, k_{0}\right) \\
\text { 1: } & \text { EVALUATE } \\
\text { 2: } & \text { PRINTRESULT } \\
\text { 3: } & \text { MORE } \\
\text { 4: } & \text { JMF end } \\
\text { 5: } & \text { FORCE } \\
& \text { proctrans }\left(\operatorname{PROC}\left(f_{1}, m_{1}, k_{1}\right)\right) \\
& \\
& \text { proctrans }\left(\operatorname{PROC}\left(f_{n}, m_{n}, k_{n}\right)\right) \\
\text { obj: } & \text { BACKTRACK lastffail } \\
& \text { exptrans } \left.\text { (OBJECTIVE }\left(k_{0}\right)\right) \\
\text { last_fail: } & \text { PRINTFAILURE } \\
\text { end: } & \text { STOP. }
\end{aligned}
$$

The first code generates a task node for the objective, starts its evaluation by EVALUATE, and prints the result of the program after a successful evaluation. If the programmer asks for more solutions, the FORCE-instruction is executed and the task of the objective is forced to backtrack.

After this preliminary code, the translation of the procedures follows. This translation is done using the proctrans scheme. Finally, code for the objective is produced. The BACKTRACK command stores the label to which to backtrack in case of a failure. The scheme exptrans produces code for the evaluation of the objective. If this evaluation fails finally this is reported by the PRINTFAILURE command.

For a function symbol $f$ with program-arity $m$ and defining rules

$$
f t_{i 1} \ldots t_{i m}:=\text { body }_{i}(1 \leq i \leq r)
$$

the following code will be generated by the scheme proctrans:

$$
\begin{aligned}
& \text { BACKTRACK label } \\
& \text { ruletrans }\left(f t_{11} \ldots t_{1 m}:=\text { body }_{1}\right) \\
& \text { label }_{1}: \text { UNDO } \\
& \text { BACKTRACK label } \\
& \text { ruletrans }\left(f t_{21} \ldots t_{2 m}:=\text { body }_{2}\right) \\
& \text { label }_{2}: \text { UNDO } \\
& \vdots \\
& \text { abel }_{r-1}: \text { UNDO } \\
& \text { BACKTRACK label } l_{r} \\
& \text { ruletrans }\left(f t_{r 1} \ldots t_{r m}:=\text { body }_{r}\right) \\
& \text { label }_{r}: \text { UNDO } \\
& \text { FAILRET }
\end{aligned}
$$


The defining rules of a function symbol are tested in their textual ordering. If all rules fail, the FAILRET command is used to force the predecessor of a task to backtrack.

The translation of each rule consists of code for the unification of the arguments of the function application with the terms on the left hand side of the rule and code for the evaluation of the body. For a rule

$$
f t_{1} \ldots t_{m}:=\text { body }
$$

the following code will be produced by the scheme ruletrans:

$$
\begin{aligned}
& \text { LOAD } 1 \\
& \text { unifytrans }\left(t_{1}\right) \\
& \vdots \\
& \text { LOAD m } \\
& \text { unifytrans }\left(t_{m}\right) \\
& \text { exptrans (body) } \\
& \text { RET }
\end{aligned}
$$

The following translation schemes are used:

- unifytrans : Term $\Sigma \rightarrow \mathcal{B A M}$-Code generates code, which unifies an argument of the actual task with the corresponding term on the left hand side of a rule.

unifytrans uses the scheme

- graphtrans : Term $\Sigma \rightarrow \mathcal{B A M}$-Code to produce code for the construction of a graph for a term, that has to be bound to an unbound variable in an argument of the actual task.

- exptrans: $\operatorname{Exp}_{\Sigma} \rightarrow \mathcal{B A M}$-Code produces code, which evaluates an expression to normal form (in particular the right hand side of a rule).

We will not go further into the general details of the code generation. In figure 2 the translation of the map example (see section 2) is given. The map example contains only one procedure with two rules. The code for the first rule - map $F[]:=[]$ - causes the following actions. First the label "nextrule" is stored which is used when the rule fails or backtracking is forced by the user. Then, the arguments of the actual task are loaded onto the stack and unified with the corresponding terms of the rule. To unify the first argument with the variable $F$, a simple UNIFYVAR instruction is sufficient. The unification of the second argument is more complicated. First it is checked (by UNIFYCONST), whether the argument is the constructor nil. If this is the case, the next instruction is executed, which causes a jump to the code for the right hand side. All arguments have now been unified with the corresponding terms on the left hand side of the rule, and hence, the rule is applicable.

If the argument starts with a constructor different from nil, the rule cannot be applied and a jump to the command referenced by the backtrack label (nextrule) is executed to try the next rule.

Otherwise, the argument must be an unbound variable. To apply the rule, this variable must be bound to the constructor nil. Hence, the UNIFYCONST instruction causes a jump to the code (starting at bindlab) which installs this binding. This code consists of a CONSTRNODE instruction, which leaves a pointer to the desired constructor node on the stack, and a BIND instruction, which overwrites the unbound variable by a variable node which points to this constructor node. The first and third possible actions of the UNIFYCONST command correspond to the read and write mode of the WAM [Warren 83 ] respectively.

The right hand side of the first rule is very simple. A constructor node with tag nil representing the empty list is produced (by CONSTRNODE) as the result, and control is given back to the calling task.

The code for the second rule - map $F[X \mid X s]:=[F X \mid$ map $F$ Xs] - starts with an UNDOinstruction that deletes the bindings that have been produced by the first rule. Then, the backtracking label "fail_lab" is stored in the actual task node. After this, the arguments of the actual task are 


\begin{tabular}{|c|c|c|c|}
\hline $\begin{array}{l}\text { bindlab: } \\
\text { continue: } \\
\text { nextrule: }\end{array}$ & $\begin{array}{l}\left.\text { NODE (obj, } 0,0, k_{0}\right) \\
\text { EVALUATE } \\
\text { PRINTRESULT } \\
\text { MORE } \\
\text { JMF end } \\
\text { FORCE } \\
\text { BACKTRACK nextrule } \\
\text { LOAD 1 } \\
\text { UNIFYVAR 1 } \\
\text { LOAD } 2 \\
\text { UNIFYCONST (nil, } 0, \text { bindlab) } \\
\text { JMP continue } \\
\text { CONSTRNODE (nil, } 0 \text { ) } \\
\text { BIND } \\
\text { CONSTRNODE (nil, 0) } \\
\text { RET } \\
\text { UNDO } \\
\text { BACKTRACK fail_lab } \\
\text { LOAD } 1 \\
\text { UNIFYVAR 1 } \\
\text { LOAD } 2 \\
\text { UNIFYCONST (cons, 2, bindlab2) } \\
\text { UNIFYVAR 2 } \\
\text { UNIFYVAR 3 } \\
\text { JMP continue2 } \\
\text { LOADX } 2\end{array}$ & $\begin{array}{r}\text { fail_lab: } \\
\qquad \text { obj: }\end{array}$ & $\begin{array}{l}\text { LOADX } 3 \\
\text { CONSTRNODE (cons, 2) } \\
\text { BIND } \\
\text { LOADX 1 } \\
\text { LOADX } 2 \\
\text { APPLY 1 } \\
\text { EVALUATE } \\
\text { LOADX 1 } \\
\text { LOADX } 3 \\
\text { NODE (map, 2, 2, 3) } \\
\text { EVALUATE } \\
\text { CONSTRNODE (cons, 2) } \\
\text { RET } \\
\text { UNDO } \\
\text { FAILRET } \\
\text { BACKTRACK last_fail } \\
\text { CONSTRNODE (1,0) } \\
\text { NODE (+, 1, 2, 2) } \\
\text { LOADX 1 } \\
\text { CONSTRNODE (2,0) } \\
\text { CONSTRNODE (cons, 2) } \\
\text { NODE (map, 2, 2, 3) } \\
\text { EVALUATE } \\
\text { RET } \\
\text { PRINTFAILURE } \\
\text { STOP }\end{array}$ \\
\hline
\end{tabular}

Figure 2: BAM-Code for the map Example Program

unified with the corresponding terms on the left hand side of the rule. For the first argument, this is exactly the same as before. For the second argument, it is more complicated, since now the term consists of an application of the binary constructor cons to two variables. If the second argument points at a constructor node for the binary constructor cons, the UNIFYCONST command now leaves pointers to both substructures of this argument on the stack. They are unified with the corresponding parts of the terms on the left hand side of the rule (here using UNIFYVAR commands). If the second argument is an unbound variable, this variable is bound to the appropriate constructor term (by the four commands following bindlab2).

The translation of the body of the rule starts at label continue2. First, the local variable $F$ is applied to the local variable $\mathrm{X}$, and the resulting task is evaluated. Furthermore, a task for map with the local variables $\mathrm{F}$ and $\mathrm{Xs}$ as arguments is created and evaluated. Both results are combined by a node for the binary constructor cons which points at them. Finally control is given back to the calling task by RET.

If the second and last rule also fails, a jump to the command referenced by the current backtrack label "fail_lab" is performed. All bindings produced by the rule are deleted and backtracking is initiated.

The code for the procedure map is followed by the translation of the objective. This translation is done analogously to the translation of the right hand side of a rule. In fact, the objective can be seen as a definition of a nullary function. 
The execution of a $B A M$-program prog starts with the following initial configuration:

$$
\text { (prog, atp, } G_{0} \text { ) }
$$

where $G_{0}$ is a graph which contains only one node representing the initial task (referenced by atp). This initial task is an active task node with the program counter initialized to 0 and its local stack and local trail are empty.

\section{Backtracking}

This section describes and justifies the backtracking information in task nodes. The content of the backtracking information that has been described in section 3.1 can be seen in figure 3 . All this information is generated each time an EVALUATE command is executed. The local trail is a list of graph addresses indicating bound variable nodes, which have to be replaced by unbound variable nodes when an UNDO-instruction is executed. Next, there are two pointers to task nodes in the graph: a so called backtracking pointer to the 'previous brother' (the node generated previously by the father) and a so called last son pointer to the 'last son' (the last son generated by the task).

\begin{tabular}{|c|c|c|c|c|c|}
\hline \multirow{2}{*}{$\begin{array}{c}\text { local } \\
\text { trail }\end{array}$} & $\begin{array}{c}\text { backtracking } \\
\text { pointer }\end{array}$ & $\begin{array}{c}\text { last son } \\
\text { pointer }\end{array}$ & backtrack & \multicolumn{3}{|c|}{ address } & $\begin{array}{c}\text { program } \\
\text { pounter }\end{array}$ & $\begin{array}{c}\text { local } \\
\text { stack }\end{array}$ & $\begin{array}{l}\text { local } \\
\text { trail }\end{array}$ \\
\cline { 4 - 5 } & & & \multicolumn{2}{|c|}{ of the father } \\
\hline
\end{tabular}

Figure 3: Backtracking information

The backtracking pointer is used when a task fails - there is no possibility of returning a value - to select the task that must then be re-evaluated. If a task is the first son of its father the backtracking pointer is the address of the father. Otherwise the backtracking pointer points at the task node that was activated by the father node just before this task.

The last son pointer is used to initialize the backtracking pointer of newly generated subtasks. It is also used to find the task to re-evaluate in case of backtracking. Of course, at any moment, the address of the active task is the last son pointer of its father.

Using these pointers one can determine an implicit stack of nodes that reflects the order in which nodes have been activated. The control of the BABEL machine is based on the following ideas:

- A task returning with success (RET command) gives the control to the father which continues its execution.

- A task returning with failure (FAILRET command) gives the control to the previous task in the implicit stack.

The recursive description of this stack in terms of the pointers in the graph is:

- The top of the stack is the right-most bottom-most element of the tree (following the last son pointer of all the nodes, beginning with the very first task generated).

- The predecessor of each task is:

1. its father if its backtracking pointer points to the father. The task is the first son.

2. Otherwise the predecessor node is the first node of the stack below the node indicated by its backtracking pointer. The predecessor can be determined by doing one step along the backtracking pointer and then following all the last son pointers. 
The following example should clarify this description. Consider the following rules of a BABEL program

$$
\begin{aligned}
& f X:=h a((p X) \rightarrow(g X) \square(l X)) \\
& p X:=(q X) \wedge(r X) . \\
& q a:=\text { true. } \\
& q b:=\text { false. } \\
& r a:=\text { true. } \\
& g X:=k X . \\
& I X:=b . \\
& b a:=a . \\
& h a b:=a .
\end{aligned}
$$

and the objective $(f X)$.

The following picture indicates the graph structure with the different pointers:

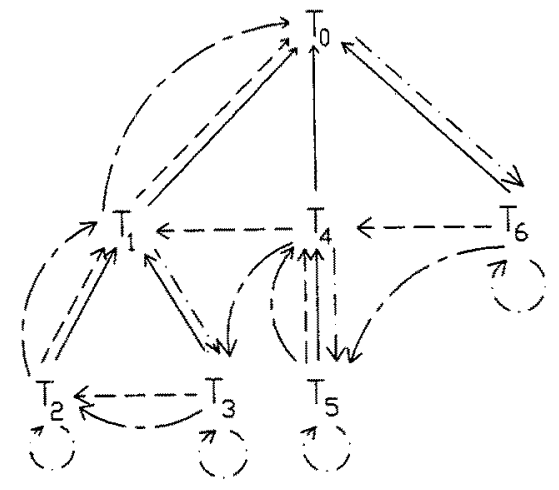

Pointers:

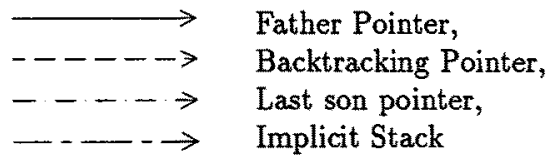

\begin{tabular}{|c||c|c|c|c|c|c|c|}
\hline Tasks & $T_{0}$ & $T_{1}$ & $T_{2}$ & $T_{3}$ & $T_{4}$ & $T_{5}$ & $T_{6}$ \\
\hline Function symbols & $f$ & $p$ & $q$ & $r$ & $g / l$ & $k$ & $h$ \\
\hline
\end{tabular}

Following the policy that a task is not removed from the stack until all its alternatives have been tried it. is clear that no answer is lost.

But some other informations for handling backtracking are needed in task nodes. The backtrack address is the address of the program where the task must continue in case of backtracking (i.e. the address of the next rule). The rest of the backtracking information is a safe copy of the state of the father: a copy of the program counter, the stack and the local trail (in fact we only need a pointer to the top of the local trail). It is used to restore the state of the father in case of backtracking, undoing all the bad decisions. The copy is made during the execution of the EVALUATE command and the restoring is made along the searching path of the next node to re-evaluate in case of backtracking.

The computation of the previous example shows why these copies are needed. The call to function f creates a task $\left(T_{0}\right)$ that is executed. In order to generate the code for the body of $f$ the first argument of the call to $h$ (constant $a$ ) is stored on the local stack. After this, a call to predicate $p$ is done which generates calls to predicates $q$ and $r$. The first value for $p$ is true binding $X$ to $a$.

The next step is to continue the execution of the BAM code for the body of $f$. The second argument of $h$ is a conditional expression. The "then" expression is selected evaluating $g$ (where evaluation of $k$ is demanded) returning $a$ to the local stack of $T_{0}$. Remember that another $a$ was stored on this stack. These two values are eliminated from the stack to construct the task ( $\left.\begin{array}{lll}h a & a\end{array}\right)$. But this task fails (there is no rule for it).

The first task to be re-evaluated is $T_{5}$ (for $k$ ). In the case that the value of this function changes, we would need to restore the local stack of $T_{0}$ to preserve the arguments of $h$ (the first $a$ on the stack). 
In our example the backtracking to task $T_{5}$ fails as well as the backtracking to $T_{4}(g)$. The condition $(p X)$ is re-evaluated. As the boolean value might change, the program counter of $T_{0}$ has to be modified to execute again the code for selecting between the "then" or the "else" branch of the conditional. The backtracking to $T_{3}(r)$ also fails, and backtracking to $T_{2}$ is needed. For this purpose it is necessary to unbind the variable $X$ because $T_{2}$ has bound it, i.e. to undo the binding of $X$ noted in the trail of $T_{2}$.

\section{Implementation}

In the implementation of the BABEL Machine we have integrated some extensions and optimizations.

The extensions suppose the inclusion of new nodes: nodes for constants (created at the beginning of the execution and shared for all the nodes that use them) and nodes for numbers. The number nodes lead to new instructions (ADD, SUB, MUL, DIV, MOD) to execute operations, +, -, *, div, mod which can be used in BABEL programs as well as integer literals. The two most important optimizations are explained in the following.

\subsection{Temporal variables}

A temporal variable of a rule is a variable that occurs on the left hand side but not inside a construction (i.e. it is directly the argument of the function). Temporal variables do not need a variable node for themselves. The argument can be used directly instead of the variable. For instance, let us consider variable $F$ in the second rule for the function map. The code used for the unification of this argument is "LOAD 1; Unifyvar 1 " and the instruction LOADX is used when $F$ is referenced in the body. We can avoid the unification of variable 1 with argument 1 and directly use the instruction LOAD 1 instead of LOADX 1 in the translation of the body. The optimization is good in memory (we need less variable nodes) and in time (we avoid the unification step).

\subsection{Disposition of task nodes}

The second optimization deals with the disposition of task nodes and is related with the reusability of "binding frames" in the Warren abstract machine.

A task node is no longer needed as soon as the existence of no more alternatives to try is noticed (execution of a FAILRET). But this situation can be found out still earlier, when the task finishes the execution of the last alternative (RET command of the last rule), because the only action that the node can execute is a return with failure. If the information stored in a task node is no longer useful, the node can be deleted from the graph and disposed. From this point of view, the essential information of a task node (w.r.t. the general behaviour of the machine) is the information about its sons (the last son pointer) and bindings that have been made during its execution (the trail). By making a compromise between time and memory efficiency, one could dispose the node of a task that finishes the execution of the last alternative if

- the task has no sons, and

- its trail is empty or

the task to be re-evaluated in case of failure is the father (its trail is appended to the trail of the father).

This optimization could be handled by a new instruction LASTRET that is used in the translation of the last rule of a function. It is very important because it allows to dispose task nodes (that need a lot of memory), makes the graph smaller and the chains inside it shorter. The performance in memory and execution time is improved because it is faster to find the node to be re-executed in case of backtracking. 


\subsection{Results}

A prototype has been implemented in OCCAM to prepare the development of parallel distributed versions. We are now developping a more serious version in $\mathrm{C}$, with the compiler written in PROLOG. With this version we will have a good measure of the real performance of the machine and compare it with functional languages and PROLOG.

However, the early results show a good behaviour in time (similar to Prolog programs) and a little bit worse in memory. Hence, the optimizations described before are very important. More optimizations need to be included in future versions, for instance a detection of purely functional computations (generating smaller task nodes by avoiding the backtracking information), special treatment of tail recursion and specific instructions for lists.

\section{Related Work}

Many contributions to the integration of the two main paradigms of declarative programming, logic and functional programming, have been investigated during the last years. [DeGroot, Lindstrom 86] collects significant papers on this field, while [Bellia, Levi 86] analyzes and classifies the main existing approaches to the integration, giving many references. Here, we restrict ourselves to comment some approaches which we find specially related to ours.

Most of the integrated logic plus functional languages proposed up to now are first order. BABEL's first order fragment [Moreno, Rodríguez 88,89], [Moreno 89] is quite related to other proposals which use systems of rewrite rules as programs and narrowing as part of the execution mechanism. As representative approaches of this kind let us mention [Reddy 85], where narrowing is proposed as the only execution mechanism, and the work by Dershowitz, Josephson and Plaisted [Dershowitz, Josephson 84], [Dershowitz, Plaisted 85], [Dershowitz 85], [Josephson, Dershowitz 86], where irrevocable (that is, not backtrackable) rewriting is used for simplification, while conditional narrowing is used for solving.

[Reddy 85] distinguishes between constructors and function symbols in the spirit of functiona] programming. He outlines a denotational semantics and a lazy narrowing strategy; based on [Hullot 80], he presents a soundness and completeness result for innermost narrowing. In first order BABEL, the declarative semantics, soundness and completeness results mentioned in section 2 above are in the spirit of least Herbrand models for Horn clause logic programs, though adapted to lazy evaluation, and much more similar to those for the language K-LEAF [Levi et al. 87] than to Reddy's results. A good discussion of completeness results for conditional narrowing from the point of view of logic plus functional programming can be found in [Giovannetti, Moiso 86].

On the other side, the work by Dershowitz, Josephson and Plaisted is more in the spirit of term rewriting theory. They view narrowing as a particular kind of the superposition operation used in the Knuth-Bendix completion procedure [Knuth, Bendix 70] and adopt an algebraic view, making no attempt to develop a constructor based denotational semantics. In [Josephson, Dershowitz 86], which discusses implementation techniques, it is suggested to use so called conditional terms to simulate conditional narrowing by unconditional rules. An equivalent technique is present in BABEL, where all rules are formally unconditional, but may have as right hand side a guarded expression, whose guard plays the role of a condition which must be narrowed to true before the body can be narrowed.

Some integrated logic plus functional languages with higher order functional programming features have also been proposed. Let us now refer to some representative cases.

[Lindstrom 87] addresses the problem of adding logical variables to a functional language, preserving lazy evaluation, concurrency opportunities, and overall determinacy. The aim is to investigate an efficient implementation on distributed architectures which provides much of the power of ANDparallel logic programming, but without support for OR-parallel search or backtracking.

[Darlington, Guo 88] have extended the functional language HOPE with absolute set abstractions [Darlington et al. 86], whose evaluation uses a kind of lazy narrowing. In this approach, logic variables 
and unification are restricted to the evaluation of absolute set abstractions. The language has been efficiently implemented.

[Reddy 87] presents a higher order functional logic language, with a denotational semantics and a narrowing based operational semantics. Explicit constructs for nondeterministic choice, existential quantification of logical variables and absolute set abstractions are introduced, and it is claimed that strict constructors are necessary to make good use of the capabilities offered by narrowing. We know of no implementation of this language.

Finally, let us mention the language IDEAL, [Bosco, Giovannetti 86], [Bellia et al. 88], which is a combination of higher order functional programming and PROLOG quite similar in expressiveness to BABEL. IDEAL is not implemented by a machine of its own, but it is instead translated to K-LEAF [Levi et al. 87] by partial evaluation with respect to an equational axiomatization of the $\lambda$-calculus; this yields clauses for a first order apply function. K-LEAF, as already discussed in the introduction, is based on Horn clause logic, and has been efficiently implemented by a conservative extension of Warren's abstract machine WAM [Warren 83] with a suspension/reactivation mechanism which emulates demand driven, lazy evaluation of function calls; cfr. [Bosco et al. 89b], [Balboni et al. 89]. This work is based on a theoretical model for emulating narrowing by SLD-resolution [Bosco et al. 88]. BABEL follows a dual approach: Its theoretical model views SLD-resolution as a particular kind of narrowing, and its implementation is achieved by extending a graph reduction machine.

\section{Conclusions and Future Work}

We have presented an implementation of a higher-order functional logic language by extension of a graph reduction machine. Logic features like unification and backtracking are embedded into this machine. The result is an abstract graph narrowing machine that may allow efficient implementations of the language, especially for functional computations.

To cope with infinite objects and non-strict functions it is necessary to change the evaluation strategy of $\mathcal{B A M}$. The development of a lazy $B A M$ which does not evaluate arguments unless they are needed, is in progress. This machine, on which we will report in a forthcoming paper, evaluates needed arguments of functions only up to head normal form. Sharing of graph nodes is more important for the lazy machine than for the innermost machine.

The main difficulty of the lazy BABEL machine is that the way in which a functional implementation treats lazy evaluation is not quite adequate for a functional logic language. To make a brief discussion we could say that a rule is applied to a subexpression $\left(f M_{1} \ldots M_{n}\right)$ following these steps:

1. Demanded arguments by the rule are evaluated until they have head normal form (i.e. there is a constructor at the root).

2. The matching between the head of the rule and the subexpression is tried. If it fails, another rule is applied.

3. The body of the rule is evaluated.

In order to use this approach in BABEL we need to take into account that if an argument does not unify with the corresponding term in the lhs of the rule this does not imply that the rule is not applicable. Another evaluation of the argument could give a new result that unifies with the term. Hence, the utilization of the same mechanism is not possible and it needs to be modified.

Another important research subject is the development of a parallel BABEL machine. In order to simplify the parallelization of $B A \mathcal{M}$, we have chosen a very decentralized structure for $B A M$. Since we are interested in using large networks of processors, we prefer a loosely coupled architecture, which consists of several processors with local memory communicating by exchanging messages. Tightly coupled architectures are not considered since their number of processors is limited. 
The parallel $B A M$ will have a structure similar to the parallel abstract machine PAM [Loogen et al. 89], which has been developped for the parallel implementation of functional languages on a loosely coupled network of processors. Each processing unit of the PAM contains a communication unit and a reduction unit. The communication units are responsible for the exchange of messages, while each reduction unit represents a sequential graph reducer that has been extended for the integration in a parallel machine. In the parallel $\mathcal{B A M}$ each reduction unit is replaced by a narrowing unit, which is an analogous extension of the sequential $\mathcal{B A M}$.

The parallelization of the $B A M$ is more complicated than the parallel implementation of a functional language. The reason is the occurrence of side effects caused by logical variables. Thus, more synchronization between parallel processes is needed. In the literature, AND- and OR-parallel implementations of logic programs are investigated. AND-parallelism can be seen as a parallel execution of the arguments of a function. In PROLOG this function is the AND-operation, in BABEL arbitrary functions can be used (as in functional languages). OR-parallelism uses a parallel execution of the different rules for each function symbol.

We are currently working on an AND- and on an OR-parallel version of the $B A M$, that will be implemented on a transputer system (like the PAM).

\section{References}

[Balboni et al. 89] G.P.Balboni, P.G.Bosco, C.Cecchi, R.Melen, C.Moiso, G.Sofi: Implementation of a Parallel Logic Plus Functional Language, in: P.Treleaven (ed.), Parallel Computers: Object Oriented, Functional and Logic, Wiley 1989.

[Bellia, Levi 86] M. Bellia, G. Levi: The Relation between Logic and Functional Languages, Journal of Logic Programming, Vol.3, 1986, 217-236.

[Bellia et al. 88] M.Bellia, P.G.Bosco, E.Giovannetti, C.Moiso, C.Palamidessi: A two level approach to logic and functional programming, CSELT Technical Reports - Vol. XVI, No. 5, August 1988.

[Bosco, Giovannetti 86] P.G.Bosco, E.Giovannetti: IDEAL: An Ideal DEductive Applicative Language, IEEE Symp. on Logic Programming 1986, IEEE Comp. Soc. Press, 89-94.

[Bosco et al. 88] P.G. Bosco, E. Giovannetti, C. Moiso: Narrowing versus SLD-resolution, Theoretical Computer Science 59, 1988, 3-23.

[Bosco et al. 89] P.G.Bosco, C.Cecchi, E.Giovannetti, C.Moiso and C.Palamidessi: Using resolution for a sound and efficient integration of logic and functional programming, in: J. de Bakker (ed.), Languages for parallel architectures: Design, semantics, implementation models, Wiley 1989.

[Bosco et al. 89b] P.G.Bosco, C.Cecchi, C.Moiso: An extension of WAM for K-LEAF: A WAM-based compilation of conditional narrowing, Int. Conf. on Logic Programming, Lisboa, 1989.

[Damas, Milner 82] L. Damas and R. Milner: Principal type schemes for functional programs, ACM Symp. on Principles of Programming Languages, 1982, 207-212.

[Darlingtion et al. 86] J.Darlington, A.J.Field and H.Pull: The unification of functional and logic languages, in: [DeGroot, Lindstrom 86], 37-70.

[Darlingtion, Guo 88] J.Darlington, Y.Guo: Narrowing and Unification in Functional Programming - An Evaluation Mechanism for Absolute Set Abstraction, Working Draft, November 1988.

[DeGroot, Lindstrom 86] D.DeGroot, G.Lindstrom (eds.): Logic Programming: Functions, Relations, Equations, Prentice Hall 1986.

[Dershowitz 85] N.Dershowitz: Computing with Rewrite Systems, Information and Control 65 ,1985, 122-157.

[Dershowitz, Josephson 84] N.Dershowitz, N.A.Josephson: Logic Programming by Completion, 2nd. Int. Conf. on Logic Programming, Uppsala, Sweden, July 1984, 313-320.

[Dershowitz, Plaisted 85] N.Dershowitz, D.A.Plaisted: Logic Programming cum Applicative Programming, IEEE Int. Symp. on Logic Programming 1985, IEEE Comp. Soc. Press, 54-66.

[van Emden, Yukawa 87] M.H.van Emden, K.Yukawa: Logic Programming with Equations, Journal of Logic Programming, Vol.4, 1987. 
[Giovannetti, Moiso 86] E.Giovannetti, C.Moiso: A completeness result for E-unification algorithms based on conditional narrowing, Workshop on Foundations of Logic and Functional Programming, Trento, Italy, Dec. 1986, LNCS 306, Springer 1986, 157-167.

[Hullot 80] J.M.Hullot: Canonical Forms and Unification, 5th Int. Conference in Automated Deduction, LNCS 87, Springer 1980, 318-334.

[Hussmann 89] H.Hussmann: Nondeterministic Algebraic Specifications and Nonconfluent Term Rewriting, Conf. on Algebraic and Logic Programming, LNCS 343, Springer 1989, 31-40.

[Josephson, Dershowitz 86] A.Josephson, N.Dershowitz: An Implementation of Narrowing: The RITE Way, Conf. on Logic Programming 1986, IEEE Comp. Soc. Press 1986, 187-197.

[Knuth, Bendix 70] D.E.Knuth, P.B.Bendix: Simple word problems in universal algebras, in "Computational Problems in Abstract Algebra", Pergamon, Oxford, U.K., 1970, 263-297.

[Kuchen et al. 89] H.Kuchen, R.Loogen, J.J. Moreno-Navarro, M.Rodríguez-Artalejo: Graph-based Implementation of a Functional Logic Language, Aachener Informatik-Berichte Nr. 89-20, RWTH Aachen, 1989.

[Levi et al. 87] G.Levi, C.Palamidessi, P.G.Bosco, E.Giovannetti and C.Moiso: A complete semantic characterization of K-LEAF, a logic language with partial functions, IEEE Int. Symp. on Logic Programming 1987, IEEE Soc. Press, 318-327.

[Lindstrom 87] G.Lindstrom: Implementing logical variables on a graph reduction architecture, Workshop on Graph Reduction, LNCS 279, Springer 1987, 382-400.

[Loogen et al. 89] R.Loogen, H.Kuchen, K.Indermark, W.Damm: Distributed Implementation of Programmed Graph Reduction, Conf. on Parallel Architectures and Languages Europe 1989, LNCS 365, Springer 1989.

[Milner 78] R. Milner: $A$ theory of type polymorphism in programming, Journal of Computer and System Sciences, 17(3), 1978.

[Moreno, Rodríguez 88] J.J.Moreno-Navarro, M.Rodriguez-Artalejo: BABEL: A functional and logic programming language based on constructor discipline and narrowing, Conf. on Algebraic and Logic Programming, LNCS 343, Springer 1989.

[Moreno, Rodríguez 89] J.J.Moreno-Navarro, M.Rodríguez-Artalejo: Logic Programming with Functions and Predicates: The Language BABEL, Technical Report DIA/89/3, Universidad Complutense, Madrid 1989, to appear in the Journal of Logic Programming.

[Moreno 89] J.J.Moreno-Navarro: Diseño, semántica e implementación de BABEL, un lenguaje que integra la programación funcional y lógica, Ph.D. Thesis, Facultad de Informática UPM, Madrid, July 1989 , (in spanish).

[Reddy 85] U.S.Reddy: Narrowing as the Operational Semantics of Functional Languages, IEEE Int. Symp. on Logic Programming, IEEE Computer Society Press, July 1985, 138-151.

[Reddy 87] U.S.Reddy: Functional Logic Languages, Part I, Workshop on Graph Reduction, LNCS 279 , Springer 1987, 401-425.

[Turner 85] D.A.Turner: Miranda: A non-strict functional language with polymorphic types, ACM Conf. on Functional Languages and Computer Architecture 1985, LNCS 201, Springer 1985, 1-16.

[Warren 83] D.H.D.Warren: An Abstract PROLOG Instruction Set, Technical Note 309, SRI International, Menlo Park, California, October 1983. 